\title{
THE EFFECT OF TIME DELAY AND HOPF BIFURCATION IN A TUMOR-IMMUNE SYSTEM COMPETITION MODEL WITH NEGATIVE IMMUNE RESPONSE
}

Abstract. We consider a system of delay differential equations modelling the tumor-immune system competition with negative immune response and three positive stationary points. The dynamics of the first two positive solutions are studied in terms of the local stability. We are particularly interested in the study of the Hopf bifurcation problem to predict the occurrence and stability of a limit cycle bifurcating from the second positive stationary point, when the delay (taken as a parameter) crosses some critical values. The results obtained provide the oscillations given by the numerical study given in Galach (2003).

1. Introduction. In this paper, we consider a model concerning the competition of tumor cells with the immune system. The modelling approach considered by many authors uses ordinary and delay differential equations (see $[17,20,24,25,28,31])$. Other authors use kinetic equations that give a complex description, at the cellular scale, in comparison with other simpler models. Kinetic models are needed to describe heterogeneity of virulence (see $[1-5,10])$.

Modelling in other fields of biology also uses kinetic equations, for instance [24] develops a kinetic theory approach to population dynamics, while [2] deals with the development of suitable general mathematical structures including a large variety of Boltzmann type models. Other authors use models stated by partial differential equations corresponding to population dynamics of cells with internal structure [29] or models based on interacting agents $[26]$.

2000 Mathematics Subject Classification: Primary 34K18.

Key words and phrases: tumor-immune system competition, negative immune response, delayed differential equations, stability, Hopf bifurcation, periodic solutions. 
The reader interested in a more complete bibliography about the evolution of a cell, and the pertinent role of cellular phenomena in directing the body towards recovery or towards illness, is referred to [16, 21]. A detailed description of virus, antivirus and body dynamics can be found in $[6,13,27,30]$.

The mathematical model under consideration was proposed in a recent paper by M. Galach [20]; it is a simple model describing tumor-immune system competition. The model consists of two ordinary differential equations with one time delay. The idea is inspired from [25]. Galach also refers to numerical results in [25] to compare them with those obtained in [20].

Our mathematical analysis is motivated by experimental and numerical results $[20,24]$. These results give evidence that the oscillating state of the tumor is more desirable, from the medical point of view, than the monotonically growing one, presumably because oscillations prolong the nonterminal phase of disease. We can ask what conditions and feedback loops make oscillations possible and whether it is possible to preserve the tumor in such oscillating state by therapeutic means for an indefinite time. This leads to the study of a model in terms of a time delay ordinary differential equation system. We believe that it makes most sense to ask qualitative questions involving behavior of the system locally with respect to time. Examples of such questions are existence and stability of fixed points and existence of Hopf bifurcation. Being interested in periodic or quasi-periodic behavior of the tumor, we investigate the model with respect to existence of a Hopf point and stability of a limit cycle.

2. Mathematical model. When an unknown tissue appears in a body, the immune system tries to identify it, and if it succeeds it attempts to eliminate it. The immune system response consists of two different interaction responses: the cellular response and the humoral response. The cellular response is carried by $\mathrm{T}$ lymphocytes. The humoral response is related to another class of cells, called B lymphocytes. The dynamics of the antitumor immune response in vivo is complicated and it is not well understood.

The immune response begins when tumor cells are identified. Then they are caught by macrophages, which can be found in all tissues in the body and circulate in the blood stream. Macrophages absorb tumor cells, eat them and release a series of cytokines which activate $\mathrm{T}$ helper cells (a subpopulation of $\mathrm{T}$ lymphocytes). These latter coordinate the counterattack. $\mathrm{T}$ helper cells can also be directly stimulated to interact with antigens. These helper cells cannot kill tumor cells, but they send urgent biochemical signals to a special type of $\mathrm{T}$ lymphocytes called natural killers (NKs). T cells begin to multiply and release other cytokines that further stimulate more $\mathrm{T}$ cells, B cells and NK cells. As the number of B cells increases, T helper cells send 
a signal to start the process of production of antibodies. Antibodies circulate in the blood and are attached to tumor cells, which implies that they are more quickly engulfed by macrophages or killed by natural killer cells. Like all $\mathrm{T}$ cells, NK cells are trained to recognize one specific type of an infected cell or a cancer cell. NK cells are lethal. They constitute a critical line of defense. A detailed description of the immune system can be found in $[4,16,30]$.

The model proposed in [25] describes the response of effector cells (ECs) (i.e. those that have been specifically activated by an antigen during the immune response and can stimulate/focus the activity of immune response leading to the elimination/destruction of the antigen) to the growth of tumor cells (TCs). This model differs from others because it takes into account the penetration of TCs by ECs, which simultaneously causes the inactivation of ECs. It is assumed that interactions between ECs and TCs in vitro can be described by the kinetic scheme shown in Fig. 1, where $E, T, C$, $E^{*}$ and $T^{*}$ are the local concentrations of ECs, TCs, EC-TC complexes, inactivated ECs, and "lethally hit" TCs, respectively, $k_{1}$ and $k_{-1}$ denote the rates of bindings of ECs to TCs and the detachment of ECs from TCs without damaging them, $k_{2}$ is the rate at which EC-TC interactions program TCs for lysis, and $k_{3}$ is the rate at which EC-TC interactions inactivate ECs.

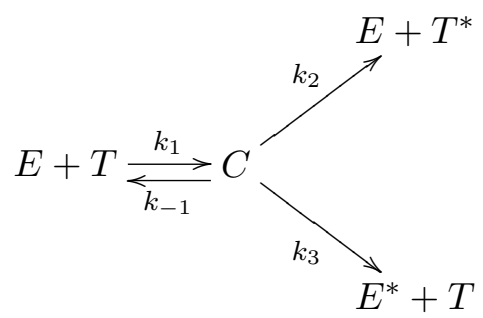

Fig. 1. Kinetic scheme describing interactions between ECs and TCs

Kuznetsov and Taylor's model is as follows:

$$
\left\{\begin{array}{l}
\frac{d E}{d t}=s+F(C, T)-d_{1} E-k_{1} E T+\left(k_{-1}+k_{2}\right) C, \\
\frac{d T}{d t}=a T(1-b T)-k_{1} E T+\left(k_{-1}+k_{3}\right) C \\
\frac{d C}{d t}=k_{1} E T-\left(k_{-1}+k_{2}+k_{3}\right) C \\
\frac{d E^{*}}{d t}=k_{3} C-d_{2} E^{*} \\
\frac{d T^{*}}{d t}=k_{2} C-d_{3} T^{*}
\end{array}\right.
$$


where $s$ is the normal (i.e. not increased by the presence of the tumor) rate of the flow of adult ECs into the tumor site, $F(C, T)$ describes the accumulation of ECs in the tumor site, $d_{1}, d_{2}$ and $d_{3}$ are the coefficients of the processes of destruction and migration for $E, E^{*}$ and $T^{*}$ respectively, $a$ is the coefficient of the maximal growth of tumor, and $b$ is the environment capacity.

In [25], it is claimed that experimental observations motivate the approximation $d C / d t \approx 0$. Therefore, it is assumed that $C \approx K E T$, where $K=k_{1} /\left(k_{2}+k_{3}+k_{-1}\right)$, and the model can be reduced to two equations which describe the behavior of ECs and TCs only. Moreover, in [20] it is suggested that the function $F$ could have the form $F(C, T)=F(E, T)=\theta E T$. Therefore, the model (2.1) takes the form

$$
\left\{\begin{array}{l}
\frac{d E}{d t}=s+\alpha_{1} E T-d E, \\
\frac{d T}{d t}=a T(1-b T)-n E T,
\end{array}\right.
$$

where $\alpha_{1}=\theta-m$, and $a, b, s$ have the same meaning as in $(2.1) ; n=K k_{2}$, $m=K k_{3}, d=d_{1}$. All coefficients except $\alpha_{1}$ are positive. The sign of $\alpha_{1}$ depends on the relation between $\theta$ and $m$. If the stimulation coefficient of the immune system exceeds the neutralization coefficient of ECs in the process of the formation of EC-TC complexes, then $\alpha_{1}>0$. We use the dimensionless form of model (2.2),

$$
\left\{\begin{array}{l}
\frac{d x}{d t}=\sigma+\omega x y-\delta x \\
\frac{d y}{d t}=\alpha y(1-\beta y)-x y,
\end{array}\right.
$$

where $x$ denotes the dimensionless density of ECs, $y$ stands for the dimensionless density of the population of TCs, $\alpha=a /\left(n T_{0}\right), \beta=b T_{0}$, $\delta=d /\left(n T_{0}\right), \sigma=s /\left(n E_{0} T_{0}\right), \omega=\alpha_{1} / n$ is immune response to the appearance of tumor cells, and $E_{0}$ and $T_{0}$ are the initial conditions. The existence, uniqueness and nonnegativity of solutions are analyzed in [20] and the the nonexistence of nonnegative periodic solution of system (2.3) is proved. The existence and stability of periodic solutions of system (2.3) are studied in [32].

For $\omega>0$ and $\alpha \delta<\sigma$, system (2.3) has one nonnegative steady state $P_{0}$, which is stable, and for $\omega>0$ and $\alpha \delta>\sigma,(2.3)$ has two possible nonnegative steady states $P_{0}$ and $P_{2}$, the first of which is unstable and the second stable (see [20]).

The delay mathematical model corresponding to (2.3) is given by the following system (see [20]): 


$$
\left\{\begin{array}{l}
\frac{d x}{d t}=\sigma+\omega x(t-\tau) y(t-\tau)-\delta x, \\
\frac{d y}{d t}=\alpha y(1-\beta y)-x y
\end{array}\right.
$$

where the parameter $\tau$ is the time delay which the immune system needs to develop for a suitable response after the recognition of nonself-cells (see [20]). Time delays in connection with tumor growth appear in many papers: see for example [7-9, 18]. The existence and uniqueness of solutions of system (2.4) for every $t>0$ are established in [20], and in the same paper it is shown that:

(1) If $\omega \geq 0$, these solutions are nonnegative for any nonnegative initial conditions (biologically realistic case).

(2) If $\omega<0$, there exist nonnegative initial conditions such that the solution becomes negative in a finite time interval.

Our goal in this paper is to consider the case (2) when the immune response is negative (i.e. $\omega<0$ ) with the following conditions: $\alpha \delta<\sigma, \omega+\beta \delta<0$ and $\alpha^{2}(\beta \delta-\omega)^{2}+4 \alpha \beta \sigma \omega>0$. We study the stability of the positive stationary points $P_{0}$ and $P_{1}$ with respect to the delay $\tau$. We establish that the Hopf bifurcation may occur at $P_{1}$ by using the delay as a parameter of bifurcation. The case (1) when the immune response is positive (i.e. $\omega>0$ ) is treated in [33], and the case $\omega<0, \alpha \delta>\sigma$ and $\alpha^{2}(\beta \delta-\omega)^{2}+4 \alpha \beta \sigma \omega>0$ is treated in [34].

This paper is organized as follows. In Section 3, we establish some results on the stability of the possible steady states (trivial and nontrivial) of the delay system (2.4). The existence of a critical value of the delay at which the nontrivial steady state $P_{1}$ changes stability is investigated. The main result of this paper is given in Section 4. Based on the Hopf bifurcation theorem, we show the occurrence of Hopf bifurcation as the delay crosses some critical value of the delay parameter ( $P_{1}$ is a Hopf point). In Section 5, we study the direction of Hopf bifurcation. In Section 6, we illustrate our result by a numerical example. Section 7 is devoted to a short discussion.

3. Steady states and stability for positive delays. Consider system (2.4), and suppose that $\omega<0, \alpha \delta<\sigma, \omega+\beta \delta<0$ and $\alpha^{2}(\beta \delta-\omega)^{2}+$ $4 \alpha \beta \sigma \omega>0$. Then system (2.4) has three equilibrium points $P_{0}=(\sigma / \delta, 0)$, $P_{1}=\left(x_{1}, y_{1}\right)$ and $P_{2}=\left(x_{2}, y_{2}\right)$ where

$$
\begin{array}{ll}
x_{1}=\frac{-\alpha(\beta \delta-\omega)-\sqrt{\Delta}}{2 \omega}, & y_{1}=\frac{\alpha(\beta \delta+\omega)+\sqrt{\Delta}}{2 \alpha \beta \omega}, \\
x_{2}=\frac{-\alpha(\beta \delta-\omega)+\sqrt{\Delta}}{2 \omega}, & y_{2}=\frac{\alpha(\beta \delta+\omega)-\sqrt{\Delta}}{2 \alpha \beta \omega},
\end{array}
$$


with $\Delta=\alpha^{2}(\beta \delta-\omega)^{2}+4 \alpha \beta \sigma \omega$. The linearized system around $P_{0}$ takes the form

$$
\left\{\begin{array}{l}
\frac{d x}{d t}=\omega \frac{\sigma}{\delta} y(t-\tau)-\delta x, \\
\frac{d y}{d t}=\left(\alpha-\frac{\sigma}{\delta}\right) y,
\end{array}\right.
$$

which leads to the characteristic equation

$$
W(\lambda)=\left(\lambda+\frac{\sigma}{\delta}-\alpha\right)(\lambda+\delta)
$$

Then we have the following result.

Proposition 3.1. Under the hypothesis $\alpha \delta<\sigma$, the equilibrium point $P_{0}$ is asymptotically stable for all $\tau>0$.

Proof. The characteristic equation (3.2) has two roots $\lambda_{1}=-\sigma / \delta+\alpha$ and $\lambda_{2}=-\delta$, which are independent of $\tau$. As $\alpha \delta<\sigma$, we have $\lambda_{i}<0$, $i=1,2$. From [22], the equilibrium point $P_{0}$ is asymptotically stable for all $\tau>0$.

Next, we shall study the stability of the nontrivial equilibrium point $P_{1}$. Let $u=x-x_{1}$ and $v=y-y_{1}$. By linearizing system (2.4) around $P_{1}$, we obtain the following linear system:

$$
\left\{\begin{array}{l}
\frac{d u}{d t}=\omega x_{1} v(t-\tau)-\omega y_{1} u(t-\tau)-\delta u, \\
\frac{d v}{d t}=-y_{1} u+\left(\alpha-2 \alpha \beta y_{1}-x_{1}\right) v .
\end{array}\right.
$$

The characteristic equation of system (3.3) has the form

$$
W(\lambda, \tau)=\lambda^{2}+p \lambda+r+(s \lambda+q) e^{-\lambda \tau}=0,
$$

where

$$
\begin{aligned}
& p=\delta+\alpha \beta y_{1}>0, \quad r=\delta \alpha \beta y_{1}>0, \\
& s=-\omega y_{1}>0, \quad q=\alpha \omega y_{1}\left(1-2 \beta y_{1}\right)<0 .
\end{aligned}
$$

The stability of $P_{1}$ depends on the localization of the roots of the equation $W(\lambda, \tau)=0$. We have the following theorem.

Theorem 3.1. Assume $\omega<0, \alpha \delta<\sigma, \omega+\beta \delta<0$ and $\alpha^{2}(\beta \delta-\omega)^{2}+$ $4 \alpha \beta \sigma \omega>0$. Then $P_{1}$ is unstable for $0 \leq \tau<\tau_{0}$ and asymptotically stable for $\tau>\tau_{0}$, where

$$
\tau_{0}=\frac{1}{\zeta_{0}} \arccos \left\{\frac{q\left(\zeta_{0}^{2}-r\right)-p s \zeta_{0}^{2}}{s^{2} \zeta_{0}^{2}+q^{2}}\right\}
$$

and

$$
\zeta_{0}^{2}=\frac{1}{2}\left(s^{2}-p^{2}+2 r\right)+\frac{1}{2}\left[\left(s^{2}-p^{2}+2 r\right)^{2}-4\left(r^{2}-q^{2}\right)\right]^{1 / 2} .
$$


For the proof, we need the following lemma.

Lemma 3.1 ([11]). Consider the equation

$$
\lambda^{2}+p \lambda+r+(s \lambda+q) e^{-\lambda \tau}=0,
$$

where $p, r, q$ and $s$ are real numbers. Assume that:

$\left(H_{1}\right) p+s>0$.

$\left(H_{2}\right) q+r<0$.

$\left(H_{3}\right) r^{2}-q^{2}<0$ or $\left(s^{2}-p^{2}+2 r>0\right.$ and $\left.\left(s^{2}-p^{2}+2 r\right)^{2}=4\left(r^{2}-q^{2}\right)\right)$.

When $\tau \in\left[0, \tau_{0}\right)$ equation (3.7) has at least one root with positive real part, when $\tau=\tau_{0}$ it has a pair of purely imaginary roots $\pm i \zeta_{0}$, and when $\tau>\tau_{0}$ both roots have negative real parts. Here $\tau_{0}$ and $\zeta_{0}$ are defined in Theorem 3.1.

Proof of Theorem 3.1. From the expressions for $p, q, s$ and $r$, we have $p+s>0$ and

$$
q+r=\alpha y_{1}\left(\omega+\delta \beta-2 \beta \omega y_{1}\right) .
$$

From the expression for $y_{1}$, we deduce that $q+r<0$. Therefore, the hypotheses $\left(H_{1}\right)$ and $\left(H_{2}\right)$ of Lemma 3.1 are satisfied. Then there exists at least one root of the characteristic equation (3.4) with positive real part for $\tau=0$ and the steady state $P_{2}$ is unstable for $\tau=0$. As

$$
r^{2}-q^{2}=(r-q)(r+q)=(r+q) \alpha y_{1}(2 \alpha \delta \beta+\sqrt{\Delta}),
$$

we have $r^{2}-q^{2}<0$ (because $\left.r+q<0\right)$ and the hypothesis $\left(H_{3}\right)$ of Lemma 3.1 is satisfied.

To obtain the switch of stability, one has to find the purely imaginary root of (3.4). If $\lambda=i w$ is a root of (3.4), then by considering the real and imaginary parts, $w$ satisfes the equation

$$
w^{4}-\left(s^{2}-p^{2}+2 r\right) w^{2}+\left(r^{2}-q^{2}\right)=0 .
$$

From Lemma 3.1, the unique solution of (3.8) is

$$
\zeta_{0}^{2}=\frac{1}{2}\left(s^{2}-p^{2}+2 r\right)+\frac{1}{2}\left[\left(s^{2}-p^{2}+2 r\right)^{2}-4\left(r^{2}-q^{2}\right)\right]^{1 / 2}
$$

and there exists a unique critical value

$$
\tau_{0}=\frac{1}{\zeta_{0}} \arccos \left\{\frac{q\left(\zeta_{0}^{2}-r\right)-p s \zeta_{0}^{2}}{s^{2} \zeta_{0}^{2}+q^{2}}\right\}
$$

such that the equilibrium point $P_{1}$ is unstable for $\tau \in\left[0, \tau_{0}\right)$ and asymptotically stable for $\tau>\tau_{0}$. For $\tau=\tau_{0}$ the characteristic equation (3.4) has a pair of purely imaginary roots $\pm i \zeta_{0}$.

In the next section, we will study the occurrence of Hopf bifurcation when the delay passes through the critical value $\tau=\tau_{0}$. 
4. Hopf bifurcation. Let $z(t)=(u(t), v(t))=(x(t), y(t))-\left(x_{1}, y_{1}\right)$. Then the system $(2.4)$ can be written as a functional differential equation $(\mathrm{FDE})$ in $C:=C\left([-\tau, 0], \mathbb{R}^{2}\right)$ :

$$
\frac{d z(t)}{d t}=L(\tau) z_{t}+f\left(z_{t}, \tau\right)
$$

where the linear operator $L(\tau): C \rightarrow \mathbb{R}^{2}$ and $f: C \times \mathbb{R} \rightarrow \mathbb{R}^{2}$ are given by

$$
L(\tau) \varphi=\left(\begin{array}{c}
\omega y_{1} \varphi_{1}(-\tau)+\omega x_{1} \varphi_{2}(-\tau)-\delta \varphi_{1}(0) \\
-y_{1} \varphi_{1}(0)+\left(\alpha-2 \alpha \beta y_{1}-x_{1}\right) \varphi_{2}(0)
\end{array}\right)
$$

and

$$
f(\varphi, \tau)=\left(\begin{array}{c}
\sigma+\omega \varphi_{1}(-\tau) \varphi_{2}(-\tau)+\omega x_{1} y_{1}-\delta x_{1} \\
-\alpha \beta \varphi_{2}^{2}(0)+\alpha y_{1}-\alpha \beta y_{1}^{2}-\varphi_{1}(0) \varphi_{2}(0)-x_{1} y_{1}
\end{array}\right)
$$

for $\varphi=\left(\varphi_{1}, \varphi_{2}\right) \in C$.

We now come to the main result of this paper.

Theorem 4.1. Assume $\omega<0, \alpha \delta<\sigma, \omega+\beta \delta<0, \alpha^{2}(\beta \delta-\omega)^{2}+$ $4 \alpha \beta \sigma \omega>0$ and $\delta$ close to 0 . There exists $\varepsilon_{1}>0$ such that, for $0 \leq \varepsilon<\varepsilon_{1}$, equation (4.1) has a family of periodic solutions $p(\varepsilon)$ with period $T=T(\varepsilon)$, for the parameter values $\tau=\tau(\varepsilon)$ such that $p(0)=P_{1}, T(0)=2 \pi / \zeta_{0}$ and $\tau(0)=\tau_{0}$, where $\tau_{0}$ and $\zeta_{0}$ are given respectively by (3.5) and (3.6).

Proof. We apply the Hopf bifurcation theorem presented in [22]. From the expression for $f$ in (4.1), we have

$$
f(0, \tau)=0 \quad \text { and } \quad \frac{\partial f(0, \tau)}{\partial \varphi}=0 \quad \text { for all } \tau>0,
$$

where $\partial / \partial \varphi$ represents the Fréchet derivative.

From Theorem 3.1, the characteristic equation (3.4) has a pair of simple imaginary roots $\lambda_{0}=i \zeta_{0}$ and $\bar{\lambda}_{0}=-i \zeta_{0}$ at $\tau=\tau_{0}$. From equation (3.4),

$W\left(\lambda_{0}, \tau_{0}\right)=0 \quad$ and $\quad \frac{\partial}{\partial \lambda} W\left(\lambda_{0}, \tau_{0}\right)=2 i \zeta_{0}+p+\left(s-\tau\left(i s \zeta_{0}+q\right)\right) e^{-i \zeta_{0} \tau_{0}} \neq 0$.

According to the implicit function theorem, there exists a complex function $\lambda=\lambda(\tau)$ defined in a neighborhood of $\tau_{0}$ such that $\lambda\left(\tau_{0}\right)=\lambda_{0}$ and $W(\lambda(\tau), \tau)=0$ and

$$
\lambda^{\prime}(\tau)=-\frac{\partial W(\lambda, \tau) / \partial \tau}{\partial W(\lambda, \tau) / \partial \lambda} \quad \text { for } \tau \text { in a neighborhood of } \tau_{0},
$$

that is,

$$
\lambda^{\prime}(\tau)=\frac{\lambda(s \lambda+q) e^{-\lambda \tau}}{2 \lambda+p+(s-\tau s \lambda-\tau q) e^{-\lambda \tau}} .
$$


From (3.4), (4.2) and (4.3), we obtain the following expression for $\lambda^{\prime}(\tau)$ for $\tau$ in a neighborhood of $\tau_{0}$ :

$$
\begin{aligned}
& \lambda^{\prime}(\tau) \\
& =-\lambda \frac{s \lambda^{3}+\left(s^{2} p+q\right) \lambda^{2}+(s r+p q) \lambda+q r}{\tau s \lambda^{3}+(s+\tau(s p+q)) \lambda^{2}+(2 q+\tau(s r+p q)) \lambda+p q-s r+q r} .
\end{aligned}
$$

Let $\lambda(\tau)=\kappa(\tau)+i \zeta(\tau)$ (where $\kappa$ and $\zeta$ are the real and imaginary parts of $\lambda$ respectively). From equation (4.4) we have

$$
\begin{aligned}
\left.\kappa^{\prime}(\tau)\right|_{\tau=\tau_{0}}= & \zeta_{0}^{2} \frac{s^{2} \zeta_{0}^{4}+\left(s q r(\tau-1)+2 q^{2}\right) \zeta_{0}^{2}+s r^{2}(q-s r)}{A^{2}+B^{2}} \\
& +\zeta_{0}^{2} \frac{p q^{2}(p+r)-q r(2 q+\tau(s r+p q))}{A^{2}+B^{2}}
\end{aligned}
$$

where

$$
\begin{aligned}
& A=-(s+\tau(s p+q)) \zeta_{0}^{2}+p q-s r+q r, \\
& B=-\tau s \zeta_{0}^{2}+(2 q+\tau(s r+p q)) \zeta_{0} .
\end{aligned}
$$

From the expression (3.4) for $r$, and as $\delta$ is close to $0, r$ is very small. From (4.5), we conclude that

$$
\left.\kappa^{\prime}(\tau)\right|_{\tau=\tau_{0}}>0 .
$$

Thus, the transversality condition is satisfied, which completes the proof of Theorem 4.1.

5. Direction of Hopf bifurcation. To determine the direction of Hopf bifurcation, there exist many formulas, e.g.: Hassard et al. [23] (formulas using the theory of normal forms), Faria and Magalhães [15], Diekmann [14]. In this section we follow the method presented in [14], where the direction and stability of the bifurcating branch are obtained by the Taylor expansion of the delay function $\tau$ that describes the parameter of bifurcation near the critical value $\tau_{0}$ (see Sections 3 and 4). Namely, this direction and stability are determined by the sign of the first nonzero term of Taylor expansion, i.e.

$$
\tau(\varepsilon)=\tau_{0}+\tau_{2} \varepsilon^{2}+o\left(\varepsilon^{2}\right)
$$

and the sign of $\tau_{2}$ determines whether the bifurcation is supercritical (if $\tau_{2}>0$ ) and periodic orbits exist for $\tau>\tau_{0}$, or subcritical (if $\tau_{2}<0$ ) and periodic orbits exist for $\tau<\tau_{0}$. The term $\tau_{2}$ may be calculated (see [14]) using the formula

$$
\tau_{2}=\frac{\operatorname{Re}(c)}{\operatorname{Re}\left(q D_{2} M\left(i \zeta_{0}, \tau_{0}\right) p\right)},
$$


where $M$ is the characteristic matrix of (4.1) given by

$$
M(\lambda, \tau)=\left(\begin{array}{cc}
\lambda+\omega y_{1} e^{-\lambda \tau}+\delta & -\omega x_{1} e^{-\lambda \tau} \\
y_{1} & \lambda-\alpha+2 \alpha \beta y_{1}+x_{1}
\end{array}\right),
$$

$D_{2} M\left(i \zeta_{0}, \tau_{0}\right)$ denotes the derivative of $M$ with respect to $\tau$ at the critical point $\left(i \zeta_{0}, \tau_{0}\right)$, and the constant $c$ is defined by

$$
\begin{aligned}
c= & \frac{1}{2} q D_{1}^{3} f\left(0, \tau_{0}\right)\left(P^{2}(\theta), \bar{P}(\theta)\right) \\
& +q D_{1}^{2} f\left(0, \tau_{0}\right)\left(e^{0 \cdot} M^{-1}\left(0, \tau_{0}\right) D_{1}^{2} f\left(0, \tau_{0}\right)(P(\theta), \bar{P}(\theta)), P(\theta)\right) \\
& +\frac{1}{2} q D_{1}^{2} f\left(0, \tau_{0}\right)\left(e^{2 i \zeta_{0}} \cdot M^{-1}\left(2 i \zeta_{0}, \tau_{0}\right) D_{1}^{2} f\left(0, \tau_{0}\right)(P(\theta), P(\theta)), \bar{P}(\theta)\right),
\end{aligned}
$$

where $f$ is the nonlinear part of (4.1), $D_{1}^{i} f, i=2,3$, denotes the $i$ th derivative of $f$ with respect to $\varphi, P(\theta)$ denotes the eigenvector of $A, \bar{P}(\theta)$ denotes the conjugate eigenvector, and $p$ and $q$ will be defined later.

Now, we describe all the above operators and vectors precisely. Let

$$
L:=L\left(\tau_{0}\right): C\left(\left[-\tau_{0}, 0\right], \mathbb{R}^{2}\right) \rightarrow \mathbb{R}^{2}
$$

denote the linear part of (4.1). Using the Riesz representation theorem one obtains (see [22])

$$
L \varphi=\int_{-\tau_{0}}^{0} d \eta(\theta) \varphi(\theta)
$$

where

$$
d \eta(\theta)=\left(\begin{array}{cc}
-\omega y_{1} \delta\left(\theta+\tau_{0}\right)-\delta \delta(\theta) & \omega x_{1} \delta\left(\theta+\tau_{0}\right) \\
-y_{1} \delta(\theta) & \left(\alpha-2 \alpha \beta y_{1}-x_{1}\right) \delta(\theta)
\end{array}\right)
$$

and $\delta(\cdot)$ denotes the Dirac function.

Let $A$ denote the generator of the semigroup generated by the linear part of (4.1). Then

$$
A \varphi(\theta)= \begin{cases}\frac{d \varphi}{d \theta}(\theta) & \text { for } \theta \in\left[-\tau_{0}, 0\right), \\ L \varphi & \text { for } \theta=0\end{cases}
$$

where $\varphi \in C\left(\left[-\tau_{0}, 0\right], \mathbb{R}^{2}\right)$.

To study the direction of Hopf bifurcation, one needs to calculate the second and third derivatives of the nonlinear part of (4.1):

$$
D_{1}^{2} f\left(0, \tau_{0}\right) \psi \chi=\left(\begin{array}{c}
\omega \psi_{1}\left(-\tau_{0}\right) \chi_{2}\left(-\tau_{0}\right)+\omega \psi_{2}\left(-\tau_{0}\right) \chi_{1}\left(-\tau_{0}\right) \\
-\psi_{1}(0) \chi_{2}(0)-\psi_{2}(0) \chi_{1}(0)-2 \alpha \beta \psi_{2}(0) \chi_{2}(0)
\end{array}\right)
$$

and 


$$
D_{1}^{3} f\left(0, \tau_{0}\right)=0
$$

for $\psi=\left(\psi_{1}, \psi_{2}\right), \chi=\left(\chi_{1}, \chi_{2}\right) \in C\left(\left[-\tau_{0}, 0\right], \mathbb{R}^{2}\right)$.

As $\left(i \zeta_{0}, \tau_{0}\right)$ is a solution of $(3.4), i \zeta_{0}$ is an eigenvalue of $A$ and there is an eigenvector of the form $P(\theta)=p e^{i \zeta_{0} \theta}$ and $p_{i}, i=1,2$, are complex numbers which satisfy the following system of equations:

$$
M\left(i \zeta_{0}, \tau_{0}\right) p=0 .
$$

Then one may assume

$$
p_{2}=1
$$

and calculate

$$
p_{1}=\frac{\alpha-2 \alpha \beta y_{1}-x_{1}-i \zeta_{0}}{y_{1}} .
$$

Now, consider $A^{*}$, i.e. the operator conjugate to $A, A^{*}: C\left([0,1], \mathbb{R}^{2}\right) \rightarrow \mathbb{R}^{2}$, defined by

$$
A^{*} \psi(s)= \begin{cases}-\frac{d \psi}{d s}(s) & \text { for } s \in(0,1], \\ -\int_{-\tau_{0}}^{0} \psi(-s) d \eta(s) & \text { for } s=0\end{cases}
$$

for $\psi=\left(\psi_{1}, \psi_{2}\right) \in C\left(\left[0, \tau_{0}\right], \mathbb{R}^{2}\right)$.

Let $Q(s)=q e^{i \zeta_{0} s}$ be the eigenvector for $A^{*}$ associated to the eigenvalue $i \zeta_{0}, q=\left(q_{1}, q_{2}\right)^{T}$. One needs to choose $q$ such that the inner product (see [22])

$$
\langle Q, \bar{P}\rangle=Q(0) \bar{P}(0)-\int_{-1}^{0} \int_{0}^{\theta} Q(\xi-\theta) d \eta(\theta) \bar{P}(\xi) d \xi
$$

be equal to 1 . Therefore

$$
q_{2}=0
$$

leads to

$$
q_{1}=\frac{e^{-\zeta_{0} \tau_{0}}}{X-i Y}
$$

where

$$
\begin{aligned}
& X=\frac{\alpha-2 \alpha \beta y_{1}-x_{1}}{y_{1}} \cos \left(\zeta_{0} \tau_{0}\right)+\frac{\zeta_{0}}{y_{1}} \sin \left(\zeta_{0} \tau_{0}\right)+\tau_{0} \omega\left(-\alpha+2 \alpha \beta y_{1}+2 x_{1}\right), \\
& Y=-\frac{\alpha-2 \alpha \beta y_{1}-x_{1}}{y_{1}} \sin \left(\zeta_{0} \tau_{0}\right)-\frac{\zeta_{0}}{y_{1}} \cos \left(\zeta_{0} \tau_{0}\right)+\zeta_{0} \tau_{0} \omega .
\end{aligned}
$$


From (5.6) we have

$$
\begin{aligned}
D_{1}^{2} f\left(0, \tau_{0}\right)(P(\theta), \bar{P}(\theta)) & =\left(\begin{array}{c}
2 \omega \operatorname{Re}\left(p_{1}\right) \\
-2 \operatorname{Re}\left(p_{1}\right)-2 \alpha \beta
\end{array}\right), \\
D_{1}^{2} f\left(0, \tau_{0}\right)(P(\theta), P(\theta)) & =\left(\begin{array}{c}
2 \omega p_{1} e^{-2 i \zeta_{0} \tau_{0}} \\
-2 p_{1}-2 \alpha \beta
\end{array}\right), \\
\frac{1}{2} q D_{1}^{3} f\left(0, \tau_{0}\right)\left(P^{2}(\theta), \bar{P}(\theta)\right) & =0 .
\end{aligned}
$$

From the expression for $M$, we have

$$
M^{-1}\left(0, \tau_{0}\right)=W^{-1}\left(0, \tau_{0}\right)\left(\begin{array}{cc}
-\alpha+2 \alpha \beta y_{1}+x_{1} & \omega x_{1} \\
-y_{1} & \omega y_{1}+\delta
\end{array}\right)
$$

and

(5.13) $M^{-1}\left(2 i \zeta_{0}, \tau_{0}\right)$

$=W^{-1}\left(2 i \zeta_{0}, \tau_{0}\right)\left(\begin{array}{cc}2 i \zeta_{0}-\alpha+2 \alpha \beta y_{1}+x_{1} & \omega x_{1} e^{-2 i \zeta_{0} \tau_{0}} \\ -y_{1} & 2 i \zeta_{0}+\omega y_{1} e^{-2 i \zeta_{0} \tau_{0}}+\delta\end{array}\right)$.

From (5.9), (5.10), (5.12) and (5.13), we have

(5.14) $q D_{1}^{2} f\left(0, \tau_{0}\right)\left(e^{0 \cdot} M^{-1}\left(0, \tau_{0}\right) D_{1}^{2} f\left(0, \tau_{0}\right)(P(\theta), \bar{P}(\theta)), P(\theta)\right)$

$$
=W\left(0, \tau_{0}\right)^{-1} \frac{\omega}{X^{2}+Y^{2}}[X+i Y][B+i C],
$$

where

$$
\begin{aligned}
B= & 2 \omega\left(-\alpha+2 \alpha \beta y_{1}\right) \operatorname{Re}\left(p_{1}\right)-2 \omega \alpha \beta x_{1} \\
& -\left(\omega y_{1}+\delta\right)\left(2 \operatorname{Re}\left(p_{1}\right)+2 \alpha \beta\right) \operatorname{Re}\left(p_{1}\right)-2 \omega y_{1} \operatorname{Re}\left(p_{1}\right)^{2}, \\
C= & -\left(\omega y_{1}+\delta\right)\left(2 \operatorname{Re}\left(p_{1}\right)+2 \alpha \beta\right) \operatorname{Im}\left(p_{1}\right)-2 \omega y_{1} \operatorname{Re}\left(p_{1}\right) \operatorname{Im}\left(p_{1}\right) .
\end{aligned}
$$

Then

$$
\begin{array}{r}
\operatorname{Re}\left(q D_{1}^{2} f\left(0, \tau_{0}\right)\left(e^{0 \cdot} M^{-1}\left(0, \tau_{0}\right) D_{1}^{2} f\left(0, \tau_{0}\right)(P(\theta), \bar{P}(\theta)), P(\theta)\right)\right) \\
=W\left(0, \tau_{0}\right)^{-1} \frac{\omega}{X^{2}+Y^{2}}[X B-Y C], \\
\begin{array}{r}
\frac{1}{2} q D_{1}^{2} f\left(0, \tau_{0}\right)\left(e^{2 i \zeta_{0} \cdot} M^{-1}\left(2 i \zeta_{0}, \tau_{0}\right) D_{1}^{2} f\left(0, \tau_{0}\right)(P(\theta), P(\theta)), \bar{P}(\theta)\right) \\
=\frac{\omega}{2} \frac{(H E-K F)+i(H F+K E)}{\left(X^{2}+Y^{2}\right)\left|W\left(2 i \zeta_{0}, \tau_{0}\right)\right|^{2}} .
\end{array}
\end{array}
$$

Then

$$
\begin{array}{r}
\text { (5.17) } \operatorname{Re}\left(\frac{1}{2} q D_{1}^{2} f\left(0, \tau_{0}\right)\left(e^{2 i \zeta_{0}} \cdot M^{-1}\left(2 i \zeta_{0}, \tau_{0}\right) D_{1}^{2} f\left(0, \tau_{0}\right)(P(\theta), P(\theta)), \bar{P}(\theta)\right)\right) \\
=\frac{\omega}{2} \frac{H E-K F}{\left(X^{2}+Y^{2}\right)\left|W\left(2 i \zeta_{0}, \tau_{0}\right)\right|^{2}},
\end{array}
$$


where

$$
\begin{aligned}
H= & X\left(-4 \zeta_{0}^{2}+r+q \cos \left(2 \zeta_{0} \tau_{0}\right)+2 s \zeta_{0} \sin \left(2 \zeta_{0} \tau_{0}\right)\right) \\
& -Y\left(2 p \zeta_{0}+2 s \zeta_{0} \cos \left(2 \zeta_{0} \tau_{0}\right)-q \sin \left(2 \zeta_{0} \tau_{0}\right)\right) \\
K= & Y\left(-4 \zeta_{0}^{2}+r+q \cos \left(2 \zeta_{0} \tau_{0}\right)+2 s \zeta_{0} \sin \left(2 \zeta_{0} \tau_{0}\right)\right) \\
& +X\left(2 p \zeta_{0}+2 s \zeta_{0} \cos \left(2 \zeta_{0} \tau_{0}\right)-q \sin \left(2 \zeta_{0} \tau_{0}\right)\right), \\
E= & \frac{2 \omega}{y_{1}}\left(\left(\alpha-2 \alpha \beta y_{1}-x_{1}\right)\left(-\alpha+2 \alpha \beta y_{1}\right)+2 \zeta_{0}^{2}\right) \\
& -\left(2\left|p_{1}\right|^{2}+\frac{2 \alpha \beta}{y_{1}}\left(\alpha-2 \alpha \beta y_{1}-x_{1}\right)\right)\left(\omega y_{1} \cos \left(2 \zeta_{0} \tau_{0}\right)+\delta\right) \\
& -\frac{\zeta_{0}}{y_{1}}\left(2 \zeta_{0}-\omega y_{1} \sin \left(2 \zeta_{0} \tau_{0}\right)\right)-2 \omega y_{1}\left|p_{1}\right|^{2} \cos \left(2 \zeta_{0} \tau_{0}\right)-2 \alpha \beta \omega x_{1}, \\
F= & \frac{2 \omega \zeta_{0}}{y_{1}}\left(3 \alpha-6 \alpha \beta y_{1}-2 x_{1}\right) \\
& -\left(2\left|p_{1}\right|^{2}+\frac{2 \alpha \beta}{y_{1}}\left(\alpha-2 \alpha \beta y_{1}-x_{1}\right)\right)\left(2 \zeta_{0}-\omega y_{1} \sin \left(2 \zeta_{0} \tau_{0}\right)\right) \\
& +\frac{\zeta_{0}}{y_{1}}\left(\omega y_{1} \cos \left(2 \zeta_{0} \tau_{0}\right)+\delta\right)+2 \omega y_{1}\left|p_{1}\right|^{2} \sin \left(2 \zeta_{0} \tau_{0}\right) .
\end{aligned}
$$

From the expressions for $M, P$ and $Q$, we have

$$
\begin{aligned}
q D_{2} M\left(i \zeta_{0}, \tau_{0}\right) p= & \frac{i \omega \zeta_{0}}{X^{2}+Y^{2}}\left(X\left(-\alpha+2 \alpha \beta y_{1}+2 x_{1}\right)\right. \\
& \left.-\zeta_{0} Y+i\left(Y\left(-\alpha+2 \alpha \beta y_{1}+2 x_{1}\right)+\zeta_{0} X\right)\right)
\end{aligned}
$$

Hence

$$
\operatorname{Re}\left(q D_{2} M\left(i \zeta_{0}, \tau_{0}\right) p\right)=-\omega \zeta_{0} \frac{Y\left(-\alpha+2 \alpha \beta y_{1}+2 x_{1}\right)+\zeta_{0} X}{X^{2}+Y^{2}} .
$$

From (5.11), (5.15), (5.17) and (5.19), the expression for $\tau_{2}$ is

$$
\tau_{2}=-\frac{\frac{1}{2} \frac{H E-K F}{\left|W\left(2 i \zeta_{0}, \tau_{0}\right)\right|^{2}}+W\left(0, \tau_{0}\right)^{-1}[X B-Y C]}{\zeta_{0}\left[Y\left(-\alpha+2 \alpha \beta y_{1}+2 x_{1}\right)-\zeta_{0} X\right]},
$$

and we deduce the following result:

TheOrem 5.1. Let $\tau_{2}$ be given in (5.20). Then

(a) the Hopf bifurcation occurs as $\tau$ crosses $\tau_{0}$ from left to right (supercritical Hopf bifurcation) if $\tau_{2}>0$ and from right to left (subcritical Hopf bifurcation) if $\tau_{2}<0$;

(b) the bifurcating periodic solution is stable if $\tau_{2}>0$ and unstable if $\tau_{2}<0$.

Note that Theorem 5.1 provides an explicit algorithm for detecting the direction and stability of Hopf bifurcation. 
6. Example. Let $\alpha=1.636, \beta=0.002, \sigma=0.1181, \delta=0.06$ and $\omega=$ 0.0085 (these parameter values come from medical experiments: Kuznetsov and Taylor [25] and Galach [20]). Then $\alpha \delta=0.0982<\sigma, \omega+\beta \delta=$ $-0.0084<0, \Delta=1.8574 \cdot 10^{-4}>0$ and system (2.4) becomes

$$
\left\{\begin{array}{l}
\frac{d x}{d t}=0.1181-0.0085 x(t-\tau) y(t-\tau)-0.06 x, \\
\frac{d y}{d t}=1.636 y(1-0.002 y)-x y .
\end{array}\right.
$$

The nontrivial steady state $P_{1}=\left(x_{1}, y_{1}\right)$ is given by

$$
x_{1}=1.6312, \quad y_{1}=1.4588,
$$

and the characteristic equation (3.4) becomes

$$
W(\lambda, \tau)=\lambda^{2}+p \lambda+r+(s \lambda+q) e^{-\lambda \tau}=0,
$$

where $p=0.0648, r=2.8638 \cdot 10^{-4}, s=0.0124$ and $q=-0.0202$.

It is easy to verify that the conditions of Theorem 4.1 are fulfilled, so a limit cycle bifurcation occurs when the time delay parameter $\tau$ passes through $\tau=\tau_{0}=20.3962$ where the relative eigenvalue is $\lambda_{0}=i 0.136$. Moreover, we can determine the approximate period of the closed orbit to be

$$
T=\frac{2 \pi}{\left|\lambda_{0}\right|}=46.1885 \text {. }
$$

From the expressions for $X, Y, H, K, B, C, E$ and $F$, we have

$$
\begin{array}{ll}
X=-0.5299, & B=5.31 \cdot 10^{-7}, \\
Y=-0.0248, & C=7.5667 \cdot 10^{-6}, \\
H=0.0419, & E=-0.0258, \\
K=-0.0016, & F=-0.0023 .
\end{array}
$$

Hence we deduce the value of $\tau_{2}$ :

$$
\tau_{2}=1.9985 \text {. }
$$

As $\tau_{2}$ is positive, from Theorem 5.1, we deduce that Hopf bifurcation occurs when $\tau$ crosses $\tau_{0}$ from left to right, and we have a supercritical Hopf bifurcation and the bifurcating branch of periodic solutions is stable.

7. Discussion. In [20], a numerical analysis shows that the characteristic equation (3.4) of the linearization of system (2.4) around the nontrivial steady state $P_{1}$ has a purely imaginary root for some $\tau=\tau_{0}$, and switching of stability may occur.

In this paper, we give an analytical study of stability (with respect to the time delay $\tau$ ) of the possible steady states $P_{0}$ and $P_{1}$ for the negative values of the parameter $\omega$ and we study each case separately. 
In Section 4, we prove that system (2.4) has a family of periodic solutions bifurcating from the nontrivial steady state $P_{1}$. The stability and Hopf bifurcation of the nontrivial steady state $P_{2}$ are studied in [34].

The results of this paper should hopefully improve the understanding of the qualitative properties of the description delivered by model (2.4). So far we have a description of stability properties and Hopf bifurcation analysis with a detailed analysis of the influence of delay terms.

\section{References}

[1] J. Adam and N. Bellomo, A Survey of Models for Tumor-Immune System Dynamics, Birkhäuser, Boston, 1996.

[2] L. Arlotti, N. Bellomo and E. De Angelis, Generalized kinetic (Boltzmann) models: Mathematical structures and applications, Math. Models Methods Appl. Sci. 12 (2002), 567-592.

[3] N. Bellomo and L. Preziosi, Modelling and mathematical problems related to tumor evolution and its interaction with the immune system, Math. Comput. Modelling 32 (2000), 413-452.

[4] N. Bellomo and M. Pulvirenti (eds.), Modelling in Applied Sciences: A Kinetic Theory Approach, Birkhäuser, 2000.

[5] N. Bellomo and M. Pulvirenti (eds.), Special issue on the modeling in applied sciences by methods of transport and kinetic theory, Math. Comput. Modelling 12 (2002), 909-990.

[6] R. Bürger, The Mathematical Theory of Selection, Recombination and Mutation, Wiley, 2000.

[7] M. Bodnar and U. Foryś, Behavior of solutions to Marchuk's model depending on a time delay, Int. Math. Comput. Sci. 10 (2000), 97-112.

[8] —, - Periodic dynamics in a model of immune system, Appl. Math. (Warsaw) 27 (2000), 113-126.

[9] H. M. Byrne, The effect of time delay on the dynamics of avascular tumor growth, Math. Biosci. 144 (1997), 83-117.

[10] M. A. J. Chaplain et al. (eds.), Special issue on mathematical models for the growth, development and treatment of tumors, Math. Models Methods Appl. Sci. 9 (1999).

[11] K. L. Cooke and Z. Grossman, Discrete delay, distributed delay and stability switches, J. Math. Anal. Appl. 86 (1982), 592-627.

[12] L. Desvillettes, C. Prvots and R. Ferrieres, Infinite dimensional reaction-diffusion for population dynamics, submitted.

[13] O. Diekmann and J. P. Heesterbeek, Mathematical Epidemiology of Infectious Diseases, Wiley, New York, 1999.

[14] O. Diekmann, S. Van Giles, S. Verduyn Lunel and H. Walter, Delay Equations, Springer, New York, 1995.

[15] T. Faria and L. Magalhães, Normal forms for retarded functional differential equations with parameters and applications to Hopf bifurcation, J. Differential Equations 122 (1995), 181-200.

[16] G. Forni, R. Fao, A. Santoni and L. Frati (eds.), Cytokine Induced Tumor Immunogeneticity, Academic Press, New York, 1994.

[17] U. Foryś, Marchuk's model of immune system dynamics with application to tumor growth, J. Theor. Med. 4 (2002), 85-93. 
[18] U. Foryś and M. Kolev, Time delays in proliferation and apoptosis for solid avascular tumor, preprint Inst. Appl. Math. and Mechanics, No. RW 02-10 (110), Warsaw Univ., 2002.

[19] U. Foryś and A. Marciniak-Czochra, Delay logistic equation with diffusion, in: Proc. 8th Nat. Conf. Application of Mathematics in Biology and Medicine, Lajs, 2002, $37-42$.

[20] M. Galach, Dynamics of the tumor-immune system competition-the effect of time delay, Int. J. Appl. Comput. Sci. 13 (2003), 395-406.

[21] L. Greller, F. Tobin and G. Poste, Tumor heterogeneity and progression: Conceptual foundation for modeling, Invasion and Metastasis 16 (1996), 177-208.

[22] J. K. Hale, Theory of Functional Differential Equations, Springer, New York, 1997.

[23] B. D. Hassard, N. D. Kazarinoff and Y. H. Wan, Theory and Applications of Hopf Bifurcation, Cambridge Univ. Press, 1981.

[24] D. Kirschner and J. C. Panetta, Modeling immunotherapy of the tumor-immune interaction, J. Math. Biol. 37 (1998), 235-252.

[25] V. A. Kuznetsov and M. A. Taylor, Nonlinear dynamics of immunogenic tumors: Parameter estimation and global bifurcation analysis, Bull. Math. Biol. 56 (1994), 295-321.

[26] P. L. Lollini, S. Motta, and F. Pappalardo, Modelling the immune competition, Math. Models Methods Appl. Sci. 16 (2006), 1091-1124.

[27] R. M. May and M. A. Nowak, Virus Dynamics (Mathematical Principles of Immunology and Virology), Oxford Univ. Press, 2000.

[28] H. Mayer, K. S. Zänker and U. der Heiden, A basic mathematical model of the immune response, Chaos 5 (1995), 155-161.

[29] P. Michel, Existence of solutions to the cell division eigenproblem, Math. Models Methods Appl. Sci. 16 (2006), 1125-1154.

[30] A. S. Perelson and G. Weisbuch, Immunology for physicists, Rev. Modern Phys. 69 (1997), 1219-1267.

[31] J. Waniewski and P. Zhivkov, A simple methematical model for tumor-immune system interactions, in: Proc. 8-th Nat. Conf. Application of Mathematics in Biology and Medicine, Lajs, 2002, 149-154.

[32] R. Yafia, Hopf bifurcation analysis and numerical simulations in an ODE model of the immune system with positive immune response, Nonlinear Anal. Real World Appl. 8 (2007), 1359-1369.

[33] - , Hopf bifurcation in differential equations with delay for tumor immune system competition model, SIAM J. Appl. Math. 67 (2007), 1693-1703.

[34] - , Hopf bifurcation in a delayed model for tumor-immune system competition with negative immune response, Discrete Dynamics Nature Soc. 2006, Art. ID 95296, 9 pp.

Faculté Polydisciplinaire

Université Ibn Zohr

B.P. 638, Ouarzazate, Morocco

E-mail: yafia1@yahoo.fr

Received on 8.6.200\%;

revised version on 2.6.2008 